\title{
Balkanologie
}

Balkanologie Revue d'études pluridisciplinaires

Vol. V, no 1-2 | 2001

Volume V Numéro 1-2

\section{Atmosphère d'après-guerre dans un village d'Herzégovine}

\section{Arnaud Appriou}

\section{OpenEdition}

1 Journals

\section{Édition électronique}

URL : http://journals.openedition.org/balkanologie/698

DOI : 10.4000/balkanologie.698

ISSN : 1965-0582

Éditeur

Association française d'études sur les Balkans (Afebalk)

Édition imprimée

Date de publication : 31 décembre 2001

ISSN : 1279-7952

\section{Référence électronique}

Arnaud Appriou, "Atmosphère d'après-guerre dans un village d'Herzégovine », Balkanologie [En ligne], Vol. V, $n^{\circ} 1-2 \mid 2001$, mis en ligne le 02 juin 2008, consulté le 17 décembre 2020. URL : http://

journals.openedition.org/balkanologie/698; DOI : https://doi.org/10.4000/balkanologie.698

Ce document a été généré automatiquement le 17 décembre 2020.

(c) Tous droits réservés 


\title{
Atmosphère d'après-guerre dans un village d'Herzégovine
}

\author{
Arnaud Appriou
}

1 En Bosnie-Herzégovine, la paix reste une réalité fragile, et la guerre, même si personne ne l'envisage à nouveau, occupe une place très importante dans le quotidien vécu et pensé des Bosniaques. Ainsi, à Blagaj, village d'Herzégovine où se situe notre terrain d'étude, la mémoire de la guerre se construit chaque jour, non pas sur des efforts permettant une paix durable, mais sur l'impact que la guerre a encore aujourd'hui sur la vie de tous les habitants de Bosnie-Herzégovine.

2 Cette mémoire se construit en grande partie dans les foyers, les maisons, ces cibles détruites massivement durant la guerre, et la trame de fond de cette construction mentale demeure souvent la définition ou les caractéristiques supposées de l'Autre, ou plus exactement des Autres. Ce sont là les bases des choix identitaires et des légitimations politiques de l'après-guerre.

3 Nous tenterons dans cet article de fournir des éléments facilitant la lecture de ces enjeux majeurs. Après avoir présenté brièvement le village de Blagaj, nous nous intéresserons aux fonctions actuelles de la maison pour ses habitants, ainsi qu'à leurs comportements identitaires et politiques.

\section{Blagaj, un village sans perspective économique à long terme}

4 Situé en contrebas du plateau de Nevesinje et en bordure de la plaine de Mostar (Bišće polje), le village de Blagaj s'étend largement sur le karst herzégovinien, et nous est ainsi décrit dans son guide touristique daté de 1998: "L'harmonie unique de la grandeur et de la beauté de la création divine. Pour l'homme, un riche trésor de la création archéologique, architecturale et culturelle »' De façon moins poétique, les habitants de Blagaj introduisent quant à eux la description des divers mahalas (quartiers, hameaux) en utilisant la très significative formule d'après-guerre : «c'était... ». Nous essaierons 
pour notre part de présenter ici la situation de ce village méditerranéen au début de l'année 1999.

D'après l'Organisation pour la sécurité et la coopération en Europe (OSCE), la population de la municipalité de Mostar-Sud-est (Mostar-Jugoistok), dans laquelle se situe Blagaj, était de 10911 habitants en 1991, dont 26 \% de Serbes et de Croates. A la même époque, le village lui-même était déjà quasiment mononational, puisque 1661 de ses 1804 habitants se déclaraient Musulmans (Bochniaques). Sept ans plus tard, la municipalité de Mostar-Sud-est compte 9780 habitants, essentiellement Bochniaques, $58 \%$ d'entre eux étant originaires des villes voisines, et appartenant donc à la catégorie des "déplacés" (displaced persons) ou des "réfugiés" (izbjeglice),d'après les taxinomies respectives des organisations internationales et de la population autochtone. Selon les témoignages recueillis, la plupart des Serbes et des Croates ont fui avant les bombardements du village par l'Armée populaire yougoslave (Jugoslovenska Narodna Armija - JNA) en 1992, puis par le Conseil de défense croate (Hrvatsko Vijeće Odbrane HVO) en 1993. Notons ici que les rares Serbes et Croates restés à Blagaj malgré les combats se nomment eux-mêmes "orthodoxes" et "catholiques". Il arrive à présent que des déplacés originaires de Blagaj se rendent au village afin de connaître le sort de la maison qu'ils occupaient jusqu'au début des hostilités. Chargées d'émotions et d'incertitudes, ces visites peuvent être le premier pas vers une future réinstallation.

Outre les nombreux morts (environ $15 \%$ de la population), les quatre années de guerre ont engendré à Blagaj d'importantes fluctuations démographiques dues à une succession, sur une période très courte, de plusieurs mouvements de population : civils et militaires, réfugiés des villes et des campagnes (jusqu'à 17000 au plus fort des combats). Le caractère tragique et souvent forcé de ces mouvements a participé à l'édification, de facto ou de jure, de nouvelles frontières qui perturbent aujourd'hui les déplacements quotidiens (commerciaux, scolaires, professionnels) et structurent les représentations mentales. Pour la population de Blagaj, les déplacements dans la partie dite croate de Mostar ou sur la côte Adriatique ne sont plus que des souvenirs, faute de pouvoir s'y rendre sans crainte pour sa propre sécurité. Le niveau scolaire de la jeunesse de Blagaj est également bouleversé : outre les problèmes causés par une scolarisation quasi inexistante entre 1993 et 1995, les habitants décrient souvent de façon dédaigneuse les mœurs de type "paysan» (seljak) des nouveaux arrivés. Quant aux réfugiés revenant de l'étranger, ils supportent mal le climat tendu et les destructions souvent plus importantes qu'au moment de leur départ. A ces mouvements de la population locale s'ajoutent enfin ceux des étrangers travaillant pour diverses organisations internationales, et qui, par leur visibilité souvent exubérante, diffusent un sentiment d'infériorité plutôt que de sécurité au sein de la population.

7 Ces mouvements seront amenés à se poursuivre dans un avenir proche. En effet, les autorités cantonales et internationales ont récemment prévu de construire quatre cent cinquante maisons sur la commune de Blagaj, afin de reloger des réfugiés. Ainsi, la question du plan d'urbanisation et des infrastructures publiques se trouve à présent posée, et la coexistence des autochtones et d'allogènes arrivés en masse ne se fera pas sans problème si le développement économique n'accompagne pas la croissance démographique. Il apparait donc nécessaire que les responsables politiques émettent des projets basés sur une vision économique à long terme. 
$8 \mathrm{Si}$, de prime abord, la population affirme souvent qu'il s'agit à présent de jouir de la vie et de la paix, les conversations en famille et entre amis sont quasiment toujours relatives aux richesses et à l'économie, et d'aucuns parlent de "guerre économique" pour décrire la paix des armes entre Croates et Bochniaques d'Herzégovine ${ }^{2}$. Mais cela ne doit pas masquer les clivages économiques existant dans chacun de ces deux groupes.

9 La situation économique est difficile, voire très précaire pour l'ensemble de la population de Blagaj : les revenus sont bas, sinon inexistants. La survie des habitants, et parfois leur bon niveau de vie, sont de ce fait un peu mystérieux, même pour les observateurs aguerris de l'économie bosniaque ${ }^{3}$. Les ressources des habitants de Blagaj sont en grande partie issues de l'agriculture et de l'élevage. L'agriculture est devenue possible après la guerre grâce à la réhabilitation du réseau hydraulique local par une O.N.G. espagnole, au moyen de fonds versés dans le cadre du programme de l'Office humanitaire des Communautés européennes (ECHO). Le jardin familial (bašta) sert de garde-manger, le garage d'étable et la voiture permet le transport de matériel en tout genre. Les échanges de biens alimentaires entre voisins et cousins sont courants. Ce type de pratiques économiques existait avant la guerre, mais leurs spécificités dans l'après-guerre sont leur généralisation et leur banalisation, même aux abords de grandes villes telles que Mostar.

10 Jusqu'en 1992, les habitants de Blagaj étaient dans leur grande majorité employés dans les entreprises d'Etat de Blagaj ou de Mostar. Les plus importantes sont à présent endommagées ou détruites, ou bien leur accès est interdit aux Bochniaques suite à une privatisation effectuée au bénéfice de la seule population croate de la région. Sokol et Aluminij, grandes entreprises de construction aéronautique et de production d'aluminium illustrent très bien cette économie identitaire. Parmi les hommes et les femmes âgés de 20 à 35 ans, le taux de chômage est très important et les activités professionnelles principales se trouvent dans les cafés, les épiceries et autres čevabdžinice (restauration rapide à base de viande hachée grillée). Les jeunes gens sont également la main-d'œuvre idéale pour construire ou rénover les maisons.

11 Si divers trafics existaient déjà avant la guerre en Herzégovine (Blagaj, semble-t-il, n'était pas épargné par le trafic de drogue), il reste que le conflit et le caractère évolutif des alliances militaires ont favorisé l'implantation durable de nouveaux réseaux commerciaux plus ou moins mafieux. Soudés par leur vie commune sur le front, dans les prisons militaires et durant la guerre en général, les membres de ces réseaux ne s'enrichissent pas forcément beaucoup, mais parviennent cependant à obtenir quelques privilèges, allant du logement gratuit à divers passe-droits lors d'achats de biens privatisables, en passant par des prix d'ami pour certains services. A ces réseaux d'entraide s'ajoute le réseau propre à chaque groupe domestique, important mais pas toujours très efficace. Tout cela favorise une économie réticulaire de proximité qui constitue un apport indispensable pour le maintien d'un niveau de vie supportable, dans cette région où l'espace économique commun, non pas délimité de jure par les accords de Dayton, mais accepté sur le terrain par les institutions internationales, s'est réduit comme une peau de chagrin.

12 Cette situation offre à certains l'opportunité d'investir dans des domaines certes livrés à la concurrence, mais qui s'appuient aussi sur les efforts de différenciation mis en oeuvre durant la guerre, tels que le tourisme religieux, tout aussi lucratif qu'exclusif. Le cas du pèlerinage de Medjugorje (qui fut un petit hameau) à vingt kilomètres à l'Ouest 
de Mostar, est devenu célèbre parmi les catholiques charismatiques du monde entier, sans être reconnu par le Vatican ${ }^{4}$. Depuis quelques années, certains cadres religieux musulmans tentent aussi d'élargir le pèlerinage annuel de l'Ajvatovica, effectué à Prusac (Bosnie centrale) et récemment promu par les autorités politiques et religieuses bochniaques $^{5}$, en y incluant une prière à Blagaj, devant la tombe d'un saint musulman (turbe de Saltuk Baba, à dix kilomètres à l'Est de Mostar). Les commerçants de Blagaj, plus sensibles aux difficultés économiques actuelles qu'aux croyances religieuses, ont à coeur de retrouver cette manne touristique qui existait durant la Yougoslavie titiste. Quels qu'ils soient, les touristes sont attendus, d'autant que, jusqu'à l'été 2000, les anciens "habitués" (Allemands, Autrichiens et Italiens principalement) avaient encore du mal à se sentir en sécurité sur les plages dalmates, et a fortiori au cœur de l'Herzégovine.

13 Ainsi, l'absence à court terme de perspectives touristiques et plus largement économiques favorise le développement d'activités précaires et plus ou moins légales. Celles-ci pérennisent les bénéfices des profiteurs de guerre et le poids de leur réseau sur la scène politique. Enfoncée dans ce marasme économique, la majeure partie de la population recentre dès lors ses activités sur la maison et la famille étroite.

\section{La maison ("kuća ») d'après-guerre : lieu de cicatrisation des plaies mal refermées}

14 L'analyse de la "maison villageoise archaïque" faite par Muhamed Kadić6 permet de comprendre à quel point la structure interne de la maison, et en particulier celle de la pièce principale (boravak) n'a pratiquement connu aucun changement jusqu'à notre époque. Le poêle, le sofa, le mur réservé à des étagères de rangement, l'espace central : la structure est conservée et la proportion des meubles également. La guerre, à Blagaj, n'a rien changé à cet agencement.

Les intérieurs révèlent pourtant les évolutions du mode de vie familial. Chaque génération apporte les symboles de son époque : si le poêle rappelle la génération des grands-parents, le portrait de Tito et le sofa dépliant sont propres à celle des parents. Enfin, le téléviseur, le Coran saoudien et les peluches sont les objets arrivés avec la dernière génération, et ces deux derniers spécialement durant le conflit: offerts par diverses organisations internationales, ils sont visibles dans la quasi totalité des foyers musulmans ${ }^{7}$. Le sérieux et la signification identitaire propres au livre saint musulman contrastent avec le caractère enfantin et insouciant des peluches multicolores. Cela semble refléter les deux pôles intellectuels auxquels les habitants de Blagaj portent un intérêt aujourd'hui, et les programmes télévisés regardés, qui servent à satisfaire l'attente suscitée par ces deux pôles, renforcent cette impression.

16 En plus d'être un espace matériel, la maison est un espace social dans lequel coexistent les membres de la famille réduite et du groupe domestique, où plusieurs générations d'hommes et de femmes ont des activités diverses, actes de la vie quotidienne ou fêtes, mariages et cérémonies de deuil ${ }^{8}$.

17 La guerre semble avoir largement interrompu un lien social peut-être propre à l'époque précédente : la génération. Que ce soit dans un cadre institutionnel (école, pionniers) ou non, l'appartenance à telle ou telle classe d'âge est un thème récurrent dans les discours, mais des réunions entre membres d'une même génération ne sont plus 
envisagées aujourd'hui ${ }^{9}$. Simultanément, les parents estiment être plus laxistes qu'auparavant avec leurs enfants : "Après la guerre, disent-ils, comment ne pas être tolérants avec nos enfants?». De nouvelles relations entre parents et enfants se profilent $^{10}$, sans que nous puissions dès à présent émettre des hypothèses sur le transfert de l'autorité morale et ses conséquences. Les limites géographiques et sociales $\mathrm{du}$ groupe domestique et du cercle d'amis ont également changé de manière significative durant la guerre : désormais, ces limites sont rarement les frontières de l'ex-Yougoslavie, et sont repoussées loin, parfois au-delà des océans. D'après des réfugiés partis durant les combats et revenus à Blagaj, et en particulier parmi les plus jeunes, les relations créées à l'étranger demeurent plus qu'un souvenir, c'est un réseau affectif à maintenir et à entretenir dans un second "chez soi".

18 La maison symbolise aussi l'antithèse des images associées à la guerre : destruction, humiliation, arrachement au terroir. Aux conditions économiques précaires s'ajoute en effet, dans l'après-guerre, un environnement psycho-affectif très déstabilisant. La violence des combats est exprimée de façon répétitive par un jeune homme de vingt-etun ans, parti sur le front à quinze ans, par l'expression : Luda kuća! ("Maison folle !"). Cette expression pourrait faire sourire si le sérieux du ton employé ne traduisait pas la prise de conscience d'un trouble psychologique généralisé. L'environnement en général est perçu comme instable et déréglé : un paysan affirme que « la guerre a même changé le climat». Cela témoigne d'un manque de stabilité ressenti par la population, d'un passage dans la paix pas encore concrétisé. La maison est dès lors un lieu privilégié de reconstruction personnelle. L'éducation des enfants, les règles de la vie sociale et l'auto-identification sont en grande partie négociées et transmises sous le toit familial. Aujourd'hui, beaucoup de temps s'écoule dans la maison, afin de gérer les tensions générées par la guerre.

19 Les habitants de Blagaj répètent et se répètent que « ici, il n'y a pas de vie », ou encore qu'«il n'y a pas de liberté ». Ces phrases simples, mais énoncées gravement et dont le sens est fort, reflètent un sentiment de temps suspendu. Le temps s'écoule lentement, et il est pesant pour tous. A cela s'ajoute un manque de régularité et de cadre temporel dans les actes de la vie quotidienne. Ainsi, les activités pour "aujourd'hui" sont rarement définies, et la projection dans le long terme en devient d'autant plus difficile, sauf en ce qui concerne la perspective de construire sa propre maison. L'inactivité professionnelle engendre une absence d'ordre. Seuls les enfants scolarisés ont, de ce point de vue, des journées et des semaines plus régulières.

20 Alors que le taux de chômage atteint vraisemblablement 80 à $90 \%$ de la population active à Blagaj, l'activité en général est donc présentée comme nécessaire, voire vitale. Non seulement pour occuper son esprit et son corps, mais surtout pour ne pas tomber dans l'apathie totale. Beaucoup l'expliquent de manière très consciente. Alors, la population s'occupe à la maison. Cette réaction participe du repli individuel et collectif sur le foyer familial. Les sorties à pied ou en voiture hors du village sont beaucoup plus rares qu'avant 1992, elles se font par obligation et non par choix.

21 Les actions répétées jusqu'à l'excès, telles les travaux de maçonnerie et les préparations culinaires multiples, ordonnent et stabilisent les individus. D'autres préfèrent le sport, la réparation de voiture ou la pêche. La guerre a souvent obligé les femmes et les enfants de Blagaj, ainsi que les hommes ne se rendant pas sur le front, à ne rien faire durant des jours et des nuits, se protégeant des obus. Actuellement, les "monoactivités" correspondent au besoin de chacun de réordonner tant sa psyché que son 
rythme de vie : la régularité et la répétition sont des outils, des repères, un moyen de ne pas rester bloqué sous le choc de la guerre.

L'atmosphère de guerre peut en effet produire de réelles paralysies. Comme nous l'a expliqué lors d'un entretien Marie-Christine Hamel, psychiatre française ayant exercé en Bosnie-Herzégovine, le siège durable d'une ville suscite des troubles psychomoteurs. A Blagaj, le siège a duré neuf mois. Du fait qu'aucune organisation nongouvernementale locale ou internationale n'ait offert après 1995 d'activités structurantes, celles-ci sont souvent liées aux pratiques religieuses. Nous avons ainsi remarqué que les prières sont effectuées plus volontiers à la maison qu'à la mosquée. Elles deviennent de cette façon un élément structurant, apaisant, et par conséquent une source d'identification.

La maison, lieu des soins physiques et psychologiques, peut enfin remplir une fonction curative plus directe. La médecine à domicile utilise les méthodes modernes et traditionnelles. Outre les souffrances générées par la guerre, diverses maladies sont soignées dans le cadre des activités familiales qui, dans leur ensemble, sont comparables à une catharsis de groupe. Ces pratiques révèlent non pas l'existence de compétences médicales diffuses mais le très mauvais état du réseau médical et pharmaceutique d'une part, et une aversion vis-à-vis de la médecine non-traditionnelle d'autre part. La population de Blagaj dans son ensemble n'est pas en bonne santé. Les dentitions sont très abîmées et soignées (arrachées a fortiori!) sur le bord du sofa. Le diabète est très répandu chez les personnes âgées, mais les soins demeurent quasi inexistants. La vue est globalement mauvaise, et les lunettes restent beaucoup trop chères pour être achetées : c'est probablement une des causes de divers accidents, dont les plus visibles sont les accidents de la route. Les rhumes et les grippes sont soignés avec des tisanes, du miel ou de la gelée de figues (pekmez). Il n'est pas non plus rare de rencontrer des cueilleurs dans les champs ou aux bords des routes, pérennisant l'utilisation de plantes sauvages curatives.

Mais tout n'est pas que soin à la maison: les cigarettes se succèdent, et la consommation d'alcool ne semble être freinée ni par la religion musulmane, ni même par la fonction purificatrice du ramadan. Il est vrai que la guerre, dans l'ensemble de la Bosnie-Herzégovine, paraît avoir largement favorisé la consommation d'alcool, de stupéfiants et de cigarettes : selon des professionnels de la santé exerçant en BosnieHerzégovine, cette consommation, poussée à l'extrême, engendre des paralysies et des amputations. Ces excès traduisent un besoin d'amnésie, de libération des tensions accumulées qui, dans l'atmosphère politique et sociale actuellement tendue, ne peuvent être correctement expulsées.

Ce descriptif de la maison et de ses fonctions serait lacunaire si le miroir de la guerre que sont les journaux télévisés n'était pas mentionné. En effet, tous les jours, les difficiles pacification et reconstruction de la Bosnie-Herzégovine et de l'ex-Yougoslavie dans son ensemble sont retransmises par les programmes télévisés, et rappellent à tous que le passé est peut-être l'avenir. Pour reprendre le titre d'un film sur la guerre en Bosnie-Herzégovine (Joli village, jolie flamme ${ }^{11}$ ), chaque petite flamme visible aujourd'hui rappelle tous les villages brûlés. Ces images télévisuelles sont, tout autant que la réalité environnante, ce que les habitants de Blagaj tentent de fuir entre les murs de la maison, faute de mieux ${ }^{12}$.

26 La maison est avant tout celle que l'on habite, mais il reste aussi celle qu'il a fallu quitter, et qu'on ne peut oublier. Enfin, une seule maison respire l'avenir meilleur: 
celle que l'on bâtit ${ }^{13}$. Elle est la matérialisation d'un nouveau départ pour des hommes et des femmes qui se fixent des objectifs et des impératifs financièrement peu réalistes, tels que se marier et avoir des enfants. Cela répond au souhait d'avoir une famille et une descendance, de se motiver pour chercher un emploi, ou simplement de « faire la seule chose qu'il reste à faire ici ». La construction de la maison, ou sa rénovation, revêt donc une signification particulière pour ces hommes et ces femmes. La blessure provoquée par sa perte durant le conflit est comparable à une plaie béante, et les autorités locales et internationales insistent sur le nécessaire retour de chacun dans son foyer. Mais il semble encore plus important, à court terme, que chaque famille puisse construire sa maison afin de reconstruire simultanément sa propre personne, ce nouvel être que chacun est devenu depuis 1992.

Face à l'étranger qu'est le chercheur, les habitants de Blagaj présentent très souvent la reconstruction de la maison comme le reflet de «l'amour du travail, caractéristique de tous les Bosniaques, quels que soient leur rang social et leur religion ». Cette apparente normalité cache cependant les changements intervenus depuis 1992. L'anthropologue norvégienne Tone Bringa affirme qu'avant la guerre « le travail bénévole effectué par les voisins pour les maisons individuelles était une autre forme d'échange social faisant appel à l'hospitalité entre familles et entre voisins $»^{14}$. Aujourd'hui, à Blagaj, chacun sait évaluer une maison, dire si elle est neuve ou reconstruite, et comment son propriétaire finance les travaux, mais la construction d'une maison n'implique ni réciprocité, ni relation de voisinage ou d'hospitalité. Il semble en fait que le groupe domestique constitue toujours un réseau important pour compresser les coûts de la construction, mais que de gros efforts et de longues discussions soient nécessaires pour le réactiver. En cas de succès, il permet de trouver un maître d'œuvre (majstor) et des maçons à moindre prix. Tout homme peut s'improviser maçon: c'est un des seuls moyens d'obtenir un revenu, ou du moins une activité bénévole. Les salaires sont rarement payés, mais un repas sert de rémunération minimale. Tout est fait pour réduire les dépenses, mais les marchands de matériaux de construction restent les gagnants dans la tourmente économique actuelle.

Lors de la construction, le prestige acquis en tant que combattant est également déterminant, en tant que source de privilèges officiels ou officieux. Les vétérans de Blagaj bénéficient de cette aura à Mostar et dans ses environs. La guerre a instauré de nouvelles sources de légitimité et de nouveaux passe-droits. Tone Bringa insiste sur le rôle que jouaient les relations au sein de la Ligue des communistes pour obtenir un terrain constructible ${ }^{15}$; aujourd'hui, ce même rôle est joué par le Parti de l'action démocratique (Stranka Demokratske Akcije), dont le maire de Blagaj est membre ${ }^{16}$. Une personne n'obtenant pas de permis de construire en mairie cherchera un bon contact (à Blagaj et si possible au sein de son groupe domestique) afin d'obtenir la signature du maire d'une autre façon. La légitimité et la reconnaissance dont bénéficiaient certaines familles à l'époque communiste perdurent cependant.

L'acte de construction déclenche des discussions sans fin avec les voisins et avec les passants: tout le monde s'informe, les projets sont expliqués, l'origine des moyens financiers est éclaircie... ou bien laissée volontairement dans l'ombre. Les déplacés qui construisent une maison et s'implantent de facto dans un nouveau village discutent également afin de se présenter et de connaître leurs voisins. Mais avant d'être acceptés et reconnus comme membres à part entière du village, il existe une phase transitoire, tout aussi perceptible dans le cas des anciens réfugiés revenus de l'étranger, bien 
qu'originaires du village. Pour ces derniers, il s'agit d'édifier sa maison en se réadaptant à l'environnement social, et se forger une nouvelle identité aux yeux des autres comme à leurs propres yeux.

Cela nous amène à nous interroger sur l'état actuel du komšiluk (bon voisinage), qui désigne, "en Bosnie-Herzégovine, le système de coexistence quotidienne entre les différentes communautés $\aleph^{17}$. La guerre, pourrions-nous penser, a annihilé le komšiluk en séparant systématiquement les groupes ethniques. Cependant, fondé sur la mitoyenneté et la reconnaissance des appartenances communautaires, le komšiluk a en fait conservé une certaine place à Blagaj, lorsque des personnes de religion différente se trouvent être encore voisines. Ainsi, il existe plusieurs cas où des chrétiens ont combattu au sein de l'armée bosniaque, ou vécu la guerre aux côtés des Bochniaques. Les habitants de Blagaj les considèrent en fonction de leur positionnement sur la ligne de front. Ainsi, le komšiluk n'a pas disparu pendant la guerre, même si le climat général, les déplacements de population et les discours des responsables politiques locaux ne favorisent pas sa pérennisation. Le komšiluk semble survivre, démontrant ainsi que la maison, théâtre de sa préservation, fonctionne comme un laboratoire où les relations sociales ne reflètent pas les décisions prises au sommet des partis identitaires et de l'Etat.

31 Dans le contexte de l'après-guerre, les personnes de confession musulmane (ou bien dont la famille mixte a été perçue comme telle pendant la guerre) se considèrent comme les victimes du conflit, et comme plus tolérants que les Serbes et les Croates. Cela a pénétré les consciences au point d'engendrer des histoires drôles et métaphoriques à ce sujet: l'une d'entre elles fait allusion à une fourmi qui s'est renforcée à travers les combats, se transformant alors en un puissant éléphant qui ne peut pas revenir à son état de fourmi fragile. La puissance apparaît ici comme une caractéristique du "nous". Il semble également que l'idée d'authenticité, de droiture et d'attitude irréprochable au cours du conflit soit plus importante pour l'identité du "nous" que les termes "Bosniaque", "Musulman" ou "Bochniaque", employés de façon relativement indifférente par la population.

Dans les conversations courantes, le terme le plus fréquemment utilisé est "Musulman / musulman". Mais s'agit-il alors d'un terme national ou religieux? La religion est en tout cas un élément important de l'identité, le moyen de ressentir au niveau local un fort sentiment d'appartenance. Elle a donc un rôle important dans la construction et la cohésion du "nous" actuel. Certains de nos interlocuteurs banalisent l'impact de l'islam et plus précisément du ramadan sur leurs activités, de façon à montrer la continuité et l'aspect volontaire des démonstrations de leur piété. Mais les habitants les plus âgés le disent ouvertement : dix ans auparavant, quasiment personne ne jeûnait. De plus, face au chercheur considéré comme catholique, un intellectuel de Blagaj justifie le ramadan par comparaison: "Tout comme les catholiques doivent manger du poisson le vendredi pour se purifier le ventre, les musulmans pratiquent le jeûne durant le ramadan ". Cela suggère que les rites musulmans sont pratiqués en parallèle et en opposition aux rites supposés des principaux ennemis durant la guerre : les Croates, perçus comme des catholiques pratiquants par les habitants de Blagaj.

Les représentants institutionnels de l'islam et les O.N.G. à caractère religieux ont un rôle majeur dans la transmission du savoir et de l'identité, au cours du ramadan comme pendant le reste de l'année. A Blagaj même, il s'agit du hodža et du mutevelija (gestionnaire des vakuf) ; au-delà, ce sont le mufti de Mostar et le Reis-ul- 
Ulema de Bosnie-Herzégovine, la Communauté islamique (Islamska Zajednica), organisation officielle des musulmans de Bosnie-Herzégovine, et l'association de bienfaisance Merhamet, proche du SDA. L'étude de ces institutions et de ces individus, porteurs de projets très divers, permettrait de mieux comprendre les défenseurs de l'islam, ou plutôt “des islams" en Bosnie-Herzégovine. Mais la diversité des islams présents aujourd'hui à Blagaj nous est déjà clairement révélée par les multiples modes d'expérimentations du ramadan. Plus que l'expression d'une piété en soi, le ramadan, et à travers lui l'islam, apparaissent donc comme un outil social de cristallisation du “nous", à mi-chemin entre laïcité et religiosité, nationalisme et mysticisme.

Enfin, le "nous" peut également renvoyer à la riche culture qui, selon les habitants de Blagaj, caractérise l'Herzégovine. La langue, la cuisine, le sol fertile, le climat et l'histoire représentent pour eux cette richesse. Le "nous" inclut alors toute la population de la région, absorbée dans un tout indifférencié, quels que soient la religion et le camp durant la guerre, mais exclut ceux qui habitent "après" Konjic, limite septentrionale de l'Herzégovine au-delà de laquelle se trouve la Bosnie.

Plus largement, les manifestations de l'identité sont très liées aux réalités locales, et pas forcément à ce qui se passe dans les administrations de la capitale Sarajevo. Il s'agit de manifester son existence, de la rendre publique, visible ou audible sur son lieu de vie. Durant le mois de ramadan, par exemple, une détonation se fait entendre chaque jour, provenant du point le plus élevé de Blagaj, afin de signaler l'autorisation de rompre le jeûne. A Mostar-est, les appels à la prière sont lancés au moyen de hautparleurs très puissants. En face, la nouvelle et imposante église catholique bâtie près de l'ancienne ligne de front a des cloches très puissantes. Ce besoin de rendre son identité tangible à l'Autre provoque une inévitable surenchère, qui est paradoxalement favorisée par le fait que les uns et les autres ne se côtoient presque plus à présent. Il s'agit de montrer ce qui crée le "nous" par des moyens audibles ou visibles, sans contact matériel direct. Si un tel contact est établi sans précaution, ses conséquences peuvent être violentes.

De même, les prises de position sur la situation de l'Etat diffèrent des réactions suscitées par les événements locaux, ces dernières étant souvent plus extrêmes : ainsi, chaque incident violent a l'effet d'une anamnèse collective qui vient rappeler l'existence de camps opposés. La publicité de ces incidents dans la population de Blagaj est très rapide et souvent excessive, ce qui accentue la sensation de ne toujours pas vivre en paix. Cela n'empêche pas l'existence d'un autre niveau de pensée qui pourrait être appelé idéel : dans l'esprit de chacun semblent ainsi coexister une Bosnie-Herzégovine idéelle, qui renvoie à des projets politiques très ouverts et tolérants vis-à-vis de l'Autre, et une Bosnie-Herzégovine de tous les dangers, pas forcément réelle, mais qui correspond aux images et aux récits de la guerre et de l'après-guerre. Le sens du "nous" peut donc varier en fonction des conversations et des activités, reflétant ainsi une amnésie sélective et instrumentale.

Cette sélective construction de la mémoire est effectuée par les personnes de plus de trente ans. Pour les plus jeunes, la guerre reste le moment fondamental dans la construction de leur identité. Cela n'a pas forcément pour conséquence une prise de conscience plus radicale, au contraire peut-être. Les jeunes ont le sentiment d'avoir tout perdu, d'avoir été sacrifiés sur un autel dont ils n'étaient pas les architectes. Et l'absence de perspectives professionnelles, le manque de liberté, ils n'hésitent pas à les fêter, à les défier en les fêtant. Ils ont dansé et bu dans certains bars de Blagaj ou au 
bord de la rivière Buna pour le Nouvel an (catholique), et ils se sont aussi beaucoup amusés dans l'ivresse du Bajram (fêtes de fin de ramadan).

Il est donc difficile aujourd'hui de trouver des constantes dans les discours des habitants de Blagaj sur leur auto-identification. Le "nous" est traversé par diverses tendances contradictoires. Un homme s'affirmant "musulman" dit spontanément, le soir du Bajram, en levant sa bière dans une main et en montrant de l'autre le kolo ${ }^{18}$ dansé sur un rythme endiablé dans un bar de la čaršija : «La véritable Bosnie, elle est là, c'est cela, elle n'est pas à la mosquée ! ». Ainsi, le "nous" a des réalités et des sens divers pour les habitants de Blagaj. Les constructions identitaires ont des frontières et des caractéristiques mobiles et négociables. L'enjeu actuel majeur reste la délimitation à long terme du Dedans et du Dehors, et ses conséquences. Un "nous" ayant pour fondement principal l'identité confessionnelle risquerait de perpétuer les tensions entre les groupes et au sein de chacun d'entre eux, et de pérenniser les espaces politiques et économiques actuels, malgré leur caractère non-viable.

Des actes sont susceptibles de donner une matérialité à l'identité collective : les repas en famille, la construction des maisons et la prière à la mosquée. Ils sont caractérisés par l'implication identitaire personnelle de chacun, par l'absence de l'Etat, et par la présence éventuelle d'une O.N.G.. Aux vues de la situation économique globale, la participation des O.N.G. et des institutions internationales est nécessaire; cependant, à plus long terme, elle favorise aussi la passivité des habitants et des autorités locales envers l'Etat. L'intense production identitaire des habitants de Blagaj se double donc d'une attitude politique attentiste et passive : le premier phénomène reflète leur forte identification à l'Etat, le second leur déception vis-à-vis de la réalité de l'Etat.

Ces deux phénomènes sont encore accentués par la légitimité du SDA qui, depuis la fin des combats, est quasiment inattaquable : le SDA est auréolé de sa présence à la tête de l'Etat durant le conflit, tandis que le Parti social-démocrate (ex-communiste) a plus de mal à valoriser sa participation à la défense de la Bosnie-Herzégovine. Le repli identitaire des Bochniaques ne peut se faire sans qu'ils accordent leur confiance aux représentants officiels de cette identité. Car ce repli s'accomplit à trois niveaux : autour de la famille, autour de la religion, et autour de la nation (narod). Il peut donc se traduire par la pratique du jeûne du ramadan comme par le vote en faveur du SDA. Et les diverses élections qui ont eu lieu à Blagaj entre 1996 et 2001 ont permis le renforcement par les urnes de la légitimité du SDA, malgré l'absence de résultats positifs tangibles dans l'après-guerre.

41 Les Bochniaques ont conscience d'avoir un dilemme à résoudre : assurer leur propre existence politique dans l'Etat de Bosnie-Herzégovine sans favoriser leurs ennemis politiques, les mêmes que pendant la guerre. Alors, beaucoup restent dans l'expectative, acceptant de donner leurs suffrages au SDA, et ne posant qu'à voix basse cette question à leurs dirigeants : «A présent, que fait-on? ». Les plus révoltés contre le SDA s'abstiennent le jour des élections. A cette attitude conservatrice et passive des électeurs correspond un comportement plus général de citoyens fatigués et impuissants : les privilèges des dirigeants des partis nationalistes ou des fonctionnaires des organisations internationales appartiennent-ils au même monde que la condition misérable des Bochniaques?

42 Par ailleurs, les journaux de la presse écrite et télévisuelle, et a fortiori les émissions étrangères retransmises en Bosnie-Herzégovine telles que les actualités de Voice of America ou les informations hebdomadaires du Tribunal pénal international, 
accentuent la désagréable impression qu'ont les Bochniaques d'être gouvernés par une administration étrangère non-élue. Ce sentiment de confiscation du pouvoir par les organisations internationales, associé à leur échec six ans après l'arrêt du conflit, peut être dangereux : le pouvoir et la présence physique massive de ces organisations n'est légitime qu'en référence à la période du conflit, et risquent de devenir illégitimes à présent, faute de pouvoir résorber la crise économique et politique. Même la partie de la population bochniaque qui reste modérée dans ses prises de positions identitaires sort de sa passivité et dénonce de façon plus ou moins sévère les errements de la politique internationale en Bosnie-Herzégovine.

La déception exprimée à l'égard de la SFOR (Stabilization Force de l'OTAN) est à ce propos exemplaire. Il est dit à Blagaj que « les soldats étrangers ne sont ici que pour percevoir un salaire beaucoup plus élevé que chez eux ». Les forces armées de l'OTAN perdent ainsi une grande part de leur respectabilité. Ce sentiment est renforcé à Blagaj par la proximité de magasins détaxés de la SFOR, qui ont la réputation d'être "dévalisés" par les soldats de la SFOR avant leur départ de Bosnie-Herzégovine. En outre, leurs passages quasi quotidiens dans le centre de Blagaj, sur le chemin d'un restaurant de poisson réputé et onéreux, rendent leur présence difficile à supporter ${ }^{19}$, même si elle est synonyme de rentrées de devises. Un décalage s'est instauré entre le fonctionnement des institutions internationales et le quotidien de la population locale. Souvent réduits aux yeux des Bochniaques à leur seule force économique, les fonctionnaires internationaux en général ne leur inspirent pas le désir de participer à leurs activités, mais représentent plutôt l'espoir d'un profit financier personnel, lorsqu'ils ne provoquent pas une aversion a priori.

Ainsi, l'Union européenne perd tout doucement le capital psychologique qu'elle avait gagné auprès d'un candidat naturel à l'entrée dans l'Union en ne soutenant pas correctement une Bosnie-Herzégovine demeurée dans ses ruines. Face au poids économique et politique qu'est censée avoir la dite communauté internationale, les Bochniaques ont tendance à s'effacer, donnant aux observateurs extérieurs une impression de passivité politique. Déplorant le blocage de l'économie à Blagaj, le représentant du Conseil de l'Europe à Mostar affirmait en juillet 1999 que «les élus politiques préfèrent se contenter d'être réélus et laissent aux autorités internationales le soin de prendre les décisions difficiles, contraires aux intérêts nationalistes de la classe politique au pouvoir ». Ce genre de passivité quasi volontaire se vérifie au plus haut niveau de l'Etat comme au niveau individuel, et se retrouve par exemple dans une remarque exprimée par un adolescent: «La rue est encore pleine de trous, aucune organisation ne l'a réparée ». Cette affirmation spontanée traduit une attitude politique assez répandue qui élude les responsabilités et les compétences de l'Etat bosniaque : dépossédée par la communauté internationale de la direction des affaires de l'Etat, la population finit par ne plus en parler, et vote par conséquent pour le candidat symboliquement fort et rassurant. Dans les perceptions et les propos des Bochniaques, l'Etat et la communauté internationale semblent en effet se partager les tâches : tandis que la seconde possède de facto le monopole du savoir-faire et de l'efficacité, l'Etat n'assure que la défense de l'identité. Ainsi pourrait s'expliquer la persistance de revendications en faveur de l'existence de plusieurs Etats dans les frontières actuelles de la Bosnie-Herzégovine. 


\section{NOTES}

1. Custo (̌̌.), Vodič kroz Blagaj, Mostar : Islamski Centar, 1998.

2. Dans ce contexte, l'enjeu des privatisations est majeur. Les autorités en charge de celles-ci semblent favoriser la privatisation d'anciennes grandes entreprises d'Etat sur des critères ethniques. Ainsi, le quotidien Oslobodjenje titrait le 6 juillet 1999 : «Privatisation ethnique dans le canton d'Herzégovine-Neretva : 180 entreprises dans les mains croates ».

3. Voir par exemple Habul (E.), «Pauvreté en Bosnie-Herzégovine : la survie des Bosniaques », Alternativna Informativna Mreža, 13 juillet 1999.

4. Voir Claverie (E.), « Voir apparaître : les “événements” de Medjugorje », Raisons pratiques, (2), 1991, pp. 157-176 ; Bax (M.), Medjugorje : Religion, Politics and Violence in Rural Bosnia, Amsterdam : VU University Press, 1995.

5. Voir Dimitrijević (D.), «Ajvatovica, analyse de la tentative de construction d'un mythe fondateur de l'identité bochniaque ", Annales de la Fondation Fyssen, (13), 1998, pp. 31-48.

6. Kadić (M.), Starinska seoska kuća u Bosni i Hercegovini, Sarajevo : Veselin Masleša, 1967.

7. Ceci n'est d'ailleurs pas spécifique aux foyers musulmans : chez des catholiques déplacés en Herzégovine occidentale ou près de Karlovac en Croatie, ou bien encore chez des orthodoxes de Banja Luka, les pièces principales sont aussi décorées de peluches sur les étagères, les meubles et les murs.

8. Les morts ne sont pas représentés dans le foyer : les photographies demeurent dans les tiroirs.

9. Par exemple, en Yougoslavie, avant 1991, les bacheliers d'une même classe se réunissaient tous les dix ans pour une soirée de souvenirs partagés.

10. C'est ce que montre Vuk Janić dans son court-métrage Psalm, Sarajevo-Amsterdam: Ifeta Stroil et Studio Production, 1997.

11. Lepa sela lepo gore, réalisé par Srdjan Blagojević, produit par Goran et Dragan Bjelogrlić, 1996.

12. Les refus des nombreuses demandes de visas d'émigration pour le Canada, les Etats-Unis ou l'Australie sont d'autant plus tragiques que le langage strict et juridique des autorités consulaires tranche avec la réalité quotidienne de la population en Bosnie-Herzégovine.

13. Les déplacés vivant à Blagaj ont toujours l'espoir de retrouver leur ancienne maison, détruite ou habitée par d'autres, à quelques dix ou vingt kilomètres de celle qu'ils occupent eux-mêmes, privant à leur tour une famille de sa maison. Mais il est arrivé que ceux qui ont pu reprendre possession de leur maison reconstruite aient la surprise de la voir à nouveau détruite, malgré la présence de la communauté internationale et de la police cantonale, prétendument neutre.

14. Bringa (T.), Being Muslim the Bosnian Way. Identity and Community in a Central Bosnian Village, Princeton : Princeton University Press, 1995, p.70.

15. Ibid., p. 74.

16. Le SDA est le principal parti nationaliste bochniaque. Lors des élections municipales d'avril 2000, il a obtenu 82,2 \% des voix dans la municipalité de Mostar-Sud-est.

17. Bougarel (X.), «Voisinage et crime intime », Confluences Méditerranée, (13), Hiver 1994-95, p. 74.

18. Cette danse traditionnelle pratiquée en cercle, rythmée par la musique populaire, participe à la construction du "nous" qui inclut et lie fortement des personnes à un groupe. Elle peut être interprétée comme la matérialisation dans l'espace de l'aspect centripète et exclusif qui caractérise l'affirmation d'un "nous".

19. Il est évident que le luxe que représente ce restaurant pour un Bosniaque n'en est pas un pour le ressortissant d'un Etat occidental. 
INDEX

Index géographique : Blagaj, Bosnie-Herzégovine, Herzégovine

\section{AUTEUR}

ARNAUD APPRIOU

Titulaire d'un D.E.A. en anthropologie sociale de l'EHESS-Paris 